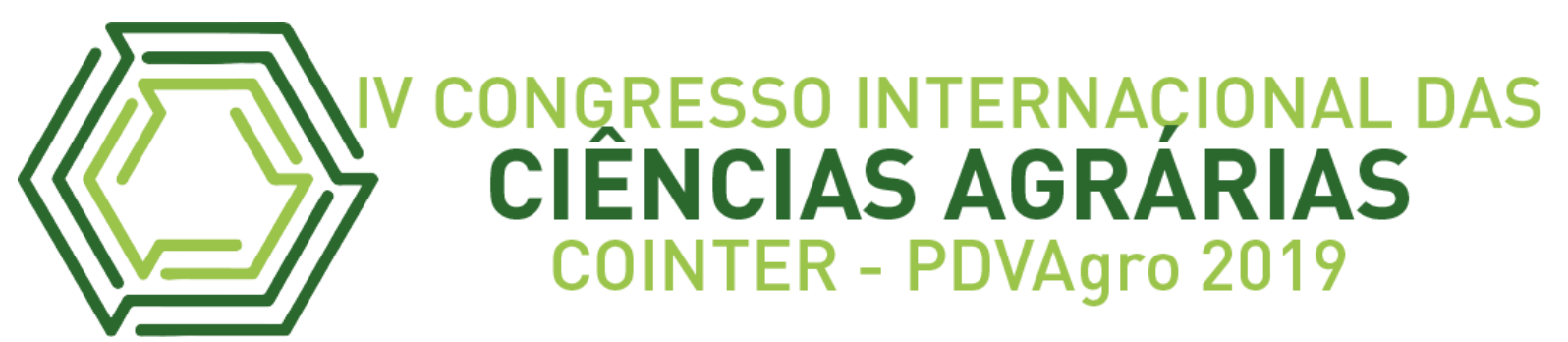

\title{
FERTIUP! - APLICATIVO DE RECOMENDAÇÕES DE ADUBAÇÃO E CALAGEM PARA PLANTAS MEDICINAIS.
}

\section{FERTIUP! - FERTIZATION AND LIMING RECOMMENDATIONS APP FOR MEDICAL PLANTS.}

\section{FERTIUP! - APP DE RECOMENDACIONES DE FERTILIZACIÓN Y ENCALHADO PARA PLANTAS MEDICINALES.}

\author{
Apresentação: Comunicação Oral \\ André Hideyoshi Afonso TANAKA ${ }^{1}$; Willian Yuki Watanabe de Lima MERA ${ }^{2}$; Alasse Oliveira da SILVA ${ }^{3}$; \\ Danilo Mesquita MELO ${ }^{4}$; Antonio Mariano Gomes da SILVA JÚNIOR ${ }^{5}$
}

DOI: https://doi.org/10.31692/2526-7701.IVCOINTERPDVAgro.2019.0044

\section{Resumo}

O cultivo e comercialização de plantas medicinais no município de Capanema é realizado por pequenos agricultores, geralmente em continuidade de uma prática herdada dos pais, sendo cultivadas conjuntamente em canteiros e hortas. Contudo, o cultivo é realizado desprovido de conhecimento tecnológico específico, sem o devido controle de qualidade na produção. Objetivou-se, assim, elaborar um aplicativo executável em smartphones com OS Android, capaz de informar o dimensionamento da área cultivada, a necessidade de calagem e adubação das principais ervas cultivadas em Capanema e região. O software intitulado "FertiUp!" foi desenvolvido na plataforma App Inventor 2, sendo o público-alvo os assessores técnicos e os produtores rurais do município de Capanema. Em sua versão 2.0.0, o FertiUp! conta com um banco de dados com 40 espécies de plantas cadastradas, 6 diferentes fontes de adubação mineral (NPK) e outras 6 fontes de adubação orgânica e o app permite que ao final o usuário possa exportar a recomendação em formato de texto para a memória interna do smartphone. Deste modo, espera-se que, após o lançamento, o FertiUp!, possa contribuir positivamente para solucionar o gargalo existente nesta modalidade de cultivo.

\footnotetext{
${ }^{1}$ Graduando em Agronomia, Universidade Federal Rural da Amazônia, UFRA/CAPANEMA, ictusandre@gmail.com

${ }^{2}$ Graduando em Agronomia, Universidade Federal Rural da Amazônia, UFRA/CAPANEMA, willian.watanabe.mera@gmail.com

${ }^{3}$ Técnico em Agronegócio (SENAR/CAPANEMA) e graduando em Agronomia pela Universidade Federal Rural da Amazônia, (UFRA/CAPANEMA), alasse.oliveira77 @ gmail.com

4 Doutor em Agronomia, professor na Universidade Federal Rural da Amazônia (UFRA/CAPANEMA), melo.agro@ hotmail.com

${ }^{5}$ Engenheiro Agrônomo pela Universidade Federal Rural da Amazônia (UFRA/CAPANEMA) e Tecnólogo em Agronegócio, marianoagronegocio@yahoo.com.br.
} 
Palavras-Chave: Nutrição de plantas, Plantas medicinais, App Inventor, Software mobile

\title{
Resumen
}

El cultivo y comercialización de plantas medicinales en el municipio de Capanema es realizado por pequeños agricultores, generalmente en la continuación de una práctica heredada de sus padres, que se cultivan juntos en parterres y huertos. Sin embargo, el cultivo se lleva a cabo sin un conocimiento tecnológico específico, sin un control de calidad adecuado en la producción. Por lo tanto, el objetivo era desarrollar una aplicación ejecutable en teléfonos inteligentes con sistema operativo Android, capaz de informar el tamaño del área cultivada, la necesidad de encalado y fertilización de las principales hierbas cultivadas en Capanema y la región. El software titulado "FertiUp!" Fue desarrollado en la plataforma App Inventor 2, con el público técnico y los productores rurales de Capanema como público objetivo. En su versión 2.0.0, FertiUp! Tiene una base de datos de 40 especies de plantas registradas, 6 fuentes diferentes de fertilizantes minerales (NPK) y 6 fuentes de fertilizantes orgánicos y la aplicación permite al usuario exportar la recomendación en formato de texto a la memoria interna. desde un teléfono inteligente Por lo tanto, se espera que después del lanzamiento, FertiUp !, pueda contribuir positivamente a resolver el cuello de botella en esta modalidad de cultivo.

Palabras Clave: Nutrición vegetal, plantas medicinales, inventor de aplicaciones, software móvil.

\begin{abstract}
The cultivation and marketing of medicinal plants in the municipality of Capanema is carried out by small farmers, usually in continuation of a practice inherited from their parents, being cultivated together in flower beds and vegetable gardens. However, cultivation is carried out without specific technological knowledge, without proper quality control in production. Thus, the objective was to develop an executable application on smartphones with Android OS, able to inform the size of the cultivated area, the need for liming and fertilization of the main herbs grown in Capanema and region. The software entitled "FertiUp!" Was developed on the App Inventor 2 platform, with the technical audience and rural producers of Capanema being the target audience. In its version 2.0.0, FertiUp! It has a database of 40 registered plant species, 6 different sources of mineral fertilizer (NPK) and 6 sources of organic fertilizer and the app allows the user to export the recommendation in text format to internal memory. from smartphone. Thus, it is expected that after launching, FertiUp!, can contribute positively to solve the bottleneck in this cultivation modality.
\end{abstract}

Keywords: Plant Nutrition, Medicinal Plants, App Inventor, Mobile Software. 


\section{Introdução}

O uso de plantas hortícolas na região amazônica, em muito, foi herdado dos povos indígenas e dos imigrantes europeus ao longo do processo de ocupação territorial (AMOROZO, 1996), sendo os grupos locais detentores de um vasto conhecimento empírico acerca dos benefícios destas plantas que destacam-se, pela qualidade nutricional conferida a dieta e possibilitam um rápido retorno econômico para o produtor (AMARO et al., 2007).

O cultivo e comercialização de hortaliças e plantas medicinais no município de Capanema, estado do Pará, é realizado, majoritariamente, por pequenos agricultores, em sistema familiar, geralmente em continuidade de uma prática herdada dos pais, sendo cultivadas conjuntamente em canteiros e hortas.

Em estudo acerca da rentabilidade da prática e das espécies mais cultivadas, Leão et al. (2016), Monteiro et al. (2017) e Silva et al. (2018) relatam um lucro mensal superior a 2 salários ( $\mathrm{R} \$ 937,00)$ com a venda das ervas, sejam eles processados ou in natura, sendo as espécies mais cultivadas pertencentes às famílias Asteraceae, Brassicaceae, Lamiaceae, Malvaceae e Zingiberaceae, havendo um comércio promissor para estas no município. Contudo, o cultivo é realizado desprovido de conhecimento tecnológico específico, sem o devido controle de qualidade na produção, por outro lado, a assessoria técnica se faz pouco presente nessas áreas, o que aumenta as dificuldades dos produtores em acessar o conhecimento necessário para uma boa produção de modo simples e objetivo.

Atualmente tal reflexo é contemplado não somente no Brasil, mas em outros países também, havendo um maior interesse da sociedade pelas hortaliças, plantas medicinais e derivados, proporcionando a abertura de mercados, geração de empregos e, no campo, a diversificação do ambiente de cultivo, enriquecendo a diversidade florística do local, contudo, o desconhecimento acerca das condições ideais de cultivo ainda persistem (MAPA, 2019).

Deste modo, objetivou-se elaborar um aplicativo de visual simples, offline, executável em smartphones com OS Android 4.0 ou superior, capaz de informar, com base nas características químicas do solo a ser cultivado, o dimensionamento da área cultivada (espaçamentos e densidade de plantio), a necessidade de calagem e adubação das principais plantas medicinais cultivadas em Capanema. 


\section{Fundamentação Teórica}

Dado que os fatores edafoclimáticos interferem diretamente no desenvolvimento e produtividade de uma espécie vegetal, é de praxe que o produtor queira controlar tais fatores a fim de conferir à planta as condições ideais ao seu crescimento, deste modo, a escolha de cultivares adaptadas e o uso racional dos insumos agrícolas hão de aumentar a produtividade da cultura. (ANTONIOLLI, 2005).

Dentre os fatores edáficos, a correção dos atributos químicos do solo por meio da calagem (neutralização do alumínio, elevação do pH e da saturação por bases), e a adubação com fertilizantes sintéticos e/ou orgânicos são práticas agronômicas cujo objetivo é favorecer o desenvolvimento da planta. Diante deste fato são propostos livros e manuais de recomendação de adubação e calagem para os diferentes estados, que podem ser feitas por cultura ou sistema de cultivo, contudo, muitos não contemplam algumas espécies frutíferas nativas da Amazônia, tornando as informações mais difíceis de ser acessadas pelas populações locais. (SBCS, 2004).

Os solos amazônicos, no que se refere aos atributos químicos, são oriundos de material mineral altamente intemperizado, dando origem a solos, que apesar de apresentar ser bem drenados, têm química pobre; sendo constituídos, majoritariamente por uma mistura de argilas e constituintes minerais, principalmente caulinita, óxidos de ferro e alumínio e silicatos, possuindo, comumente, uma baixa capacidade de troca de cátions, geralmente inferior a 17 cmolc. $\mathrm{kg}^{-1}$.

Raij et al. (2001) e Lepsch (2016) explicam que em regiões tropicais os solos são na maioria das vezes ácidos devido aos altos índices pluviométricos que lixiviam as bases (Cálcio $\left[\mathrm{Ca}^{2+}\right]$, Magnésio $\left[\mathrm{Mg}^{2+}\right]$ e Potássio $\left.\left[\mathrm{K}^{+}\right]\right)$, e pela ausência de minerais primários que reponham estas bases; de modo que, a melhor forma de corrigir estes solos é por meio das práticas de calagem e adubação à valores adequados à cultura a ser implantada.

De acordo com a Associação Brasileira de Marketing Rural e Agronegócio - AMBRA (2018) o uso de smartphones no meio rural saltou $40 \%$ nos últimos 5 anos, sendo a idade média dos usuários entrevistados de 45 anos ou superior. Dentre os sistemas operacionais mais utilizados no Brasil, o Android OS, desenvolvido pela empresa Google LLC, responde 
por cerca de $95 \%$ dos smartphones ativos no país, segundo dados anunciados por KANTAR (2018).

Em um estudo realizado pelo Serviço Brasileiro de Apoio às Micro e Pequenas Empresas (SEBRAE), foram entrevistados mais de 4 mil produtores rurais, nas vinte e sete Unidades Federativas e, obtiveram que, 34,3\% utilizam Internet via rádio e 24,3\% utilizam a banda larga móvel, 46,3\% avaliaram ainda que a qualidade do serviço no meio rural é regular, atribuindo nota 6, numa escala de 0 a 10 (SEBRAE, 2017).

Embora se tenha amplo conhecimento da nutrição de plantas e das influências das práticas de cultivo sobre a produção e rendimento dos vegetais, são poucos os manuais de adubação e calagem que contemplam as recomendações para tais e, os que contemplam, apresentam um caráter generalista; por outro lado, a divulgação recente de ensaios agronômicos em revistas científicas possui uma linguagem rebuscada e/ou apresentam-se em outro idioma o que dificulta o acesso das comunidades locais a este conhecimento.

O MIT App Inventor é uma plataforma online de programação visual "drag and drop" para desenvolver aplicativos móveis operantes em sistema Android. O App Inventor foi lançado em 2013, trazendo uma nova era na programação de softwares, com uma interface intuitiva e simples, a plataforma permite que o desenvolvedor se concentre na lógica de programação, em vez da linguagem de codificação em si (POKRESS e VEIGA, 2013).

A interface do App Inventor é extremamente simples, fundamentada na ideia de "lowfloor" e o ambiente de desenvolvimento em "high-ceiling", apresentando duas partes: uma janela "Designer" (Figura 1a), sendo a parte visual onde é possível selecionar os objetos do aplicativo e um "Blocks Editor" (Figura 1b) onde se 'escreve' o comportamento de cada

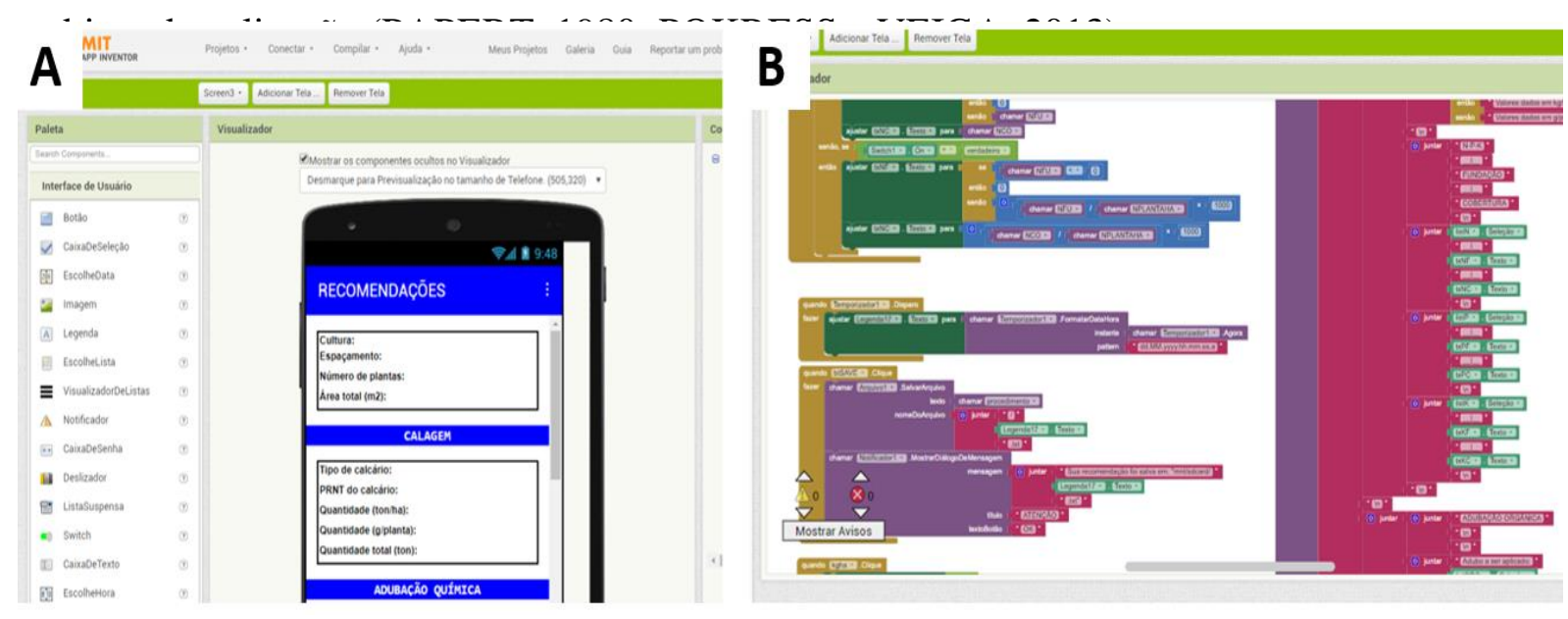




\section{Metodologia}

O software "FertiUp!" foi desenvolvido na plataforma online e gratuita App Inventor 2 (MIT, 2013), sendo utilizada a linguagem de programação JAVA fundamentada no conceito de Programação Orientada a Objetos (POO). A utilização do Java é comum entre programadores por ser multiplataforma e deter uma grande variedade de frameworks (GONÇALVES, 2010). A Programação Orientada a Objetos serve para aproximar a escrita (linhas de código) do cenário real a partir da abstração de objetos, tornando-o visível / palpável, além de favorecer o aproveitamento de trechos do código fonte e maior segurança do sistema (BERNARDI, 2011).

O procedimento para o desenvolvimento do aplicativo foi dividido em 4 etapas interdependentes, conforme metodologia proposta por Duda et al. (2015), a saber:

Etapa 1 - Caracterização do app e estruturação do banco de dados: Nessa etapa, foi definido o enfoque da pesquisa e o objeto de estudo, realizando o levantamento dos dados e referências para formar o banco de dados [em *.csv, no software Microsoft Office Excel 2016];

Etapa 2 - Arquitetura: com o banco de dados pronto, estruturou-se um fluxograma para as rotinas de cálculo a ser realizadas pelo aplicativo (Figura 2), resultando em uma versão alpha do software;

Etapa 3 - Revisão e testes: etapa onde foi verificada existência de falhas e erros de execução, sendo estes corrigidos ou adaptados. Nesse momento foi possível aferir se a proposta inicial foi atingida na elaboração do aplicativo;

Etapa 4 - Estabilização: etapa onde o aplicativo teve sua estrutura e funcionalidades validadas pelos autores/desenvolvedores, evoluindo para uma versão beta. 
FIGURA 2 - Fluxograma de execução da rotina de cálculos do FertiUp!.

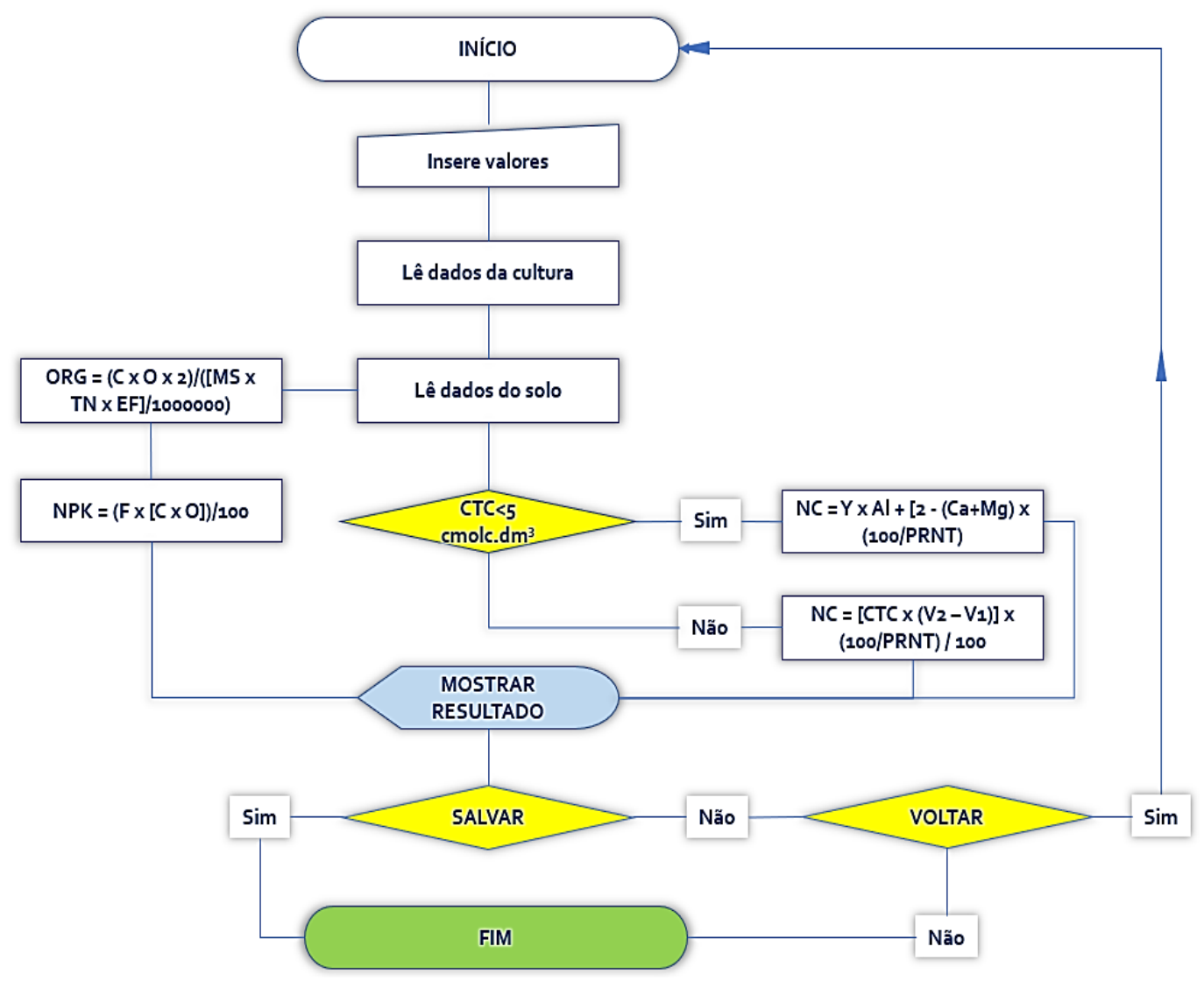

Os cálculos utilizados na construção do app foram extraídos dos manuais de adubação e calagem do estado do Pará (CRAVO et al., 2007), do Boletim de adubação 200 - IAC (AGUIAR et al., 2014) e do boletim técnico de adubação orgânica $\mathrm{N}^{\circ} 96$ - UFLA (LACERDA e SILVA, 2014), sendo os mesmos adaptados para a execução pelo aplicativo, estando dispostos nas equações $1,2,3$ e 4 .

EQUAÇÃO 1 - Necessidade de calagem via elevação da saturação de bases.

$$
\mathrm{NC}=[\mathrm{CTC} \times(\mathrm{V} 2-\mathrm{V} 1) \times(100 / \mathrm{PRNT})] / 100
$$

$\mathrm{NC}=$ Necessidade de calcário, em t/ha; 
$\mathrm{CTC}=($ capacidade de troca de cátions $)$ em cmolc/dm3;

V2 = Saturação por bases desejada;

V1 = Saturação por bases atual do solo;

PRNT $=$ Poder Relativo de Neutralização Total.

EQUAÇÃO 2 - Necessidade de calagem via elevação dos teores de Ca e Mg.

$$
\mathrm{NC}=\mathrm{Y} \times \mathrm{Al}+[2-(\mathrm{Ca}+\mathrm{Mg}) \times(100 / \mathrm{PRNT})]
$$

$\mathrm{Y}=1$ para solos arenosos;

$\mathrm{Y}=2$ para solos com textura média;

$\mathrm{Y}=3$ para solos argilosos;

$\mathrm{NC}=$ Necessidade de calcário, em t/ha;

$\mathrm{Al}=$ teor de alumínio no solo;

$\mathrm{Ca}+\mathrm{Mg}=$ teor de cálcio e magnésio no solo;

PRNT $=$ Poder Relativo de Neutralização Total.

EQUAÇÃO 3 - Necessidade de adubo orgânico em quilos por hectare.

$$
\mathrm{X}=[\mathrm{A} /(\mathrm{B} / \mathbf{1 0 0} \times \mathrm{C} / 100 \times \mathrm{D} / \mathbf{1 0 0})] \times 2
$$

$\mathrm{X}=$ Quantidade do adubo orgânico a aplicar $\left(\mathrm{kg} \mathrm{ha}^{-1}\right)$;

$\mathrm{A}=$ Quantidade do nutriente exigida $\left(\mathrm{kg} \mathrm{ha}^{-1}\right)$; 
$\mathrm{B}=$ Teor de matéria seca do fertilizante $(\%)$

$\mathrm{C}=$ Teor do nutriente na matéria seca $(\%)$

$\mathrm{D}=$ Índice de conversão $(\%)[\mathrm{N}=50 ; \mathrm{P}=60 ; \mathrm{K}=100]$

EQUAÇÃO 4 - Necessidade de fertilizante químico em quilos por hectare.

$$
Y=[(A-X) \times F] / 100
$$

$\mathrm{Y}=$ Quantidade do adubo químico a aplicar $\left(\mathrm{kg} \mathrm{ha}^{-1}\right)$;

$\mathrm{A}=$ Quantidade do nutriente exigida $\left(\mathrm{kg} \mathrm{ha}^{-1}\right)$;

$\mathrm{X}=$ Quantidade do nutriente fornecida pelo adubo orgânico $\left(\mathrm{kg} \mathrm{ha}^{-1}\right)$;

$\mathrm{F}$ = fator de conversão para cada fertilizante químico (\%).

\section{Resultados e Discussão}

Em sua versão 2.0.0, o FertiUp! conta com um banco de dados com 40 espécies de plantas medicinais cadastradas, dentre as quais: Alecrim, (ABDELAZIZ et al., 2007), açafrão (OCAMPO e VALVERDE, 2000), arnica (OLIVEIRA JÚNIOR et al., 2006), aloe vera (SARADHI, 2007), bardana (GASSI, 2006), entre outras (SOUSA et al., 2006; AZEVEDO e MOURA, 2010; LOPES, 2014); há 6 diferentes fontes de adubação mineral (NPK) e outras 6 fontes de adubação orgânica (LACERDA e SILVA, 2014; AGUIAR et al., 2014), havendo um deslizador que proporciona, em termos percentuais, as quantidades dos adubos entre orgânico e mineral, conforme a escolha do usuário. $\mathrm{O}$ aplicativo permite ainda que ao final de cada recomendação o operador possa exportar a recomendação exibida em formato de texto (*.txt) para a memória interna do smartphone, ficando disponível para acesso posterior (Figura 3).

O aplicativo permite ainda saber a quantidade a ser aplicada em área total (ton.ha ${ }^{-1}$, kg.ha ${ }^{-1}$ e em g.planta $\left.{ }^{-1}\right)$, espaçamentos e número de plantas na área total. Contudo, vale 
destacar que os erros ocorridos durante a coleta das amostras de solo, ou na inserção dos valores no app interferem diretamente no resultado da análise e na recomendação de adubação e calagem, ou seja, o laboratório de análises químicas não pode corrigir falhas decorrentes de erros na amostragem do solo, logo é fundamental que se tenha a consciência de que o uso do FertiUp! de modo algum escusa a necessidade de um técnico nesta área de atuação (solos, produção vegetal, nutrição de plantas, ...) (MOREIRA et al., 2008; TAVARES et al., 2015).

Muito embora o aplicativo ainda não tenha sido apresentado ao público até a presente data (Agosto de 2019), espera-se que o mesmo tenha uma boa receptividade pela comunidade de produtores e técnicos e que possa vir a sanar as dificuldades no cultivo destas plantas, difundindo-se, através deste software, aos técnicos e produtores o conhecimento científico existente a respeito do preparo do solo para a produção de ervas medicinais.

Reitera-se que após o lançamento, o app contará com atualizações regulares, para aumento do banco de dados e correção de erros, sendo proposto para que, em versão futura, haja uma expansão do banco de dados para mais culturas de relevante expressividade econômica no município.

FIGURA 3 - Telas do app: abertura (1), opções (2), sobre o aplicativo (3), dados do cultivo (4 e 5), dados do local (6), recomendações (7), recomendação exportada (8 e 9).

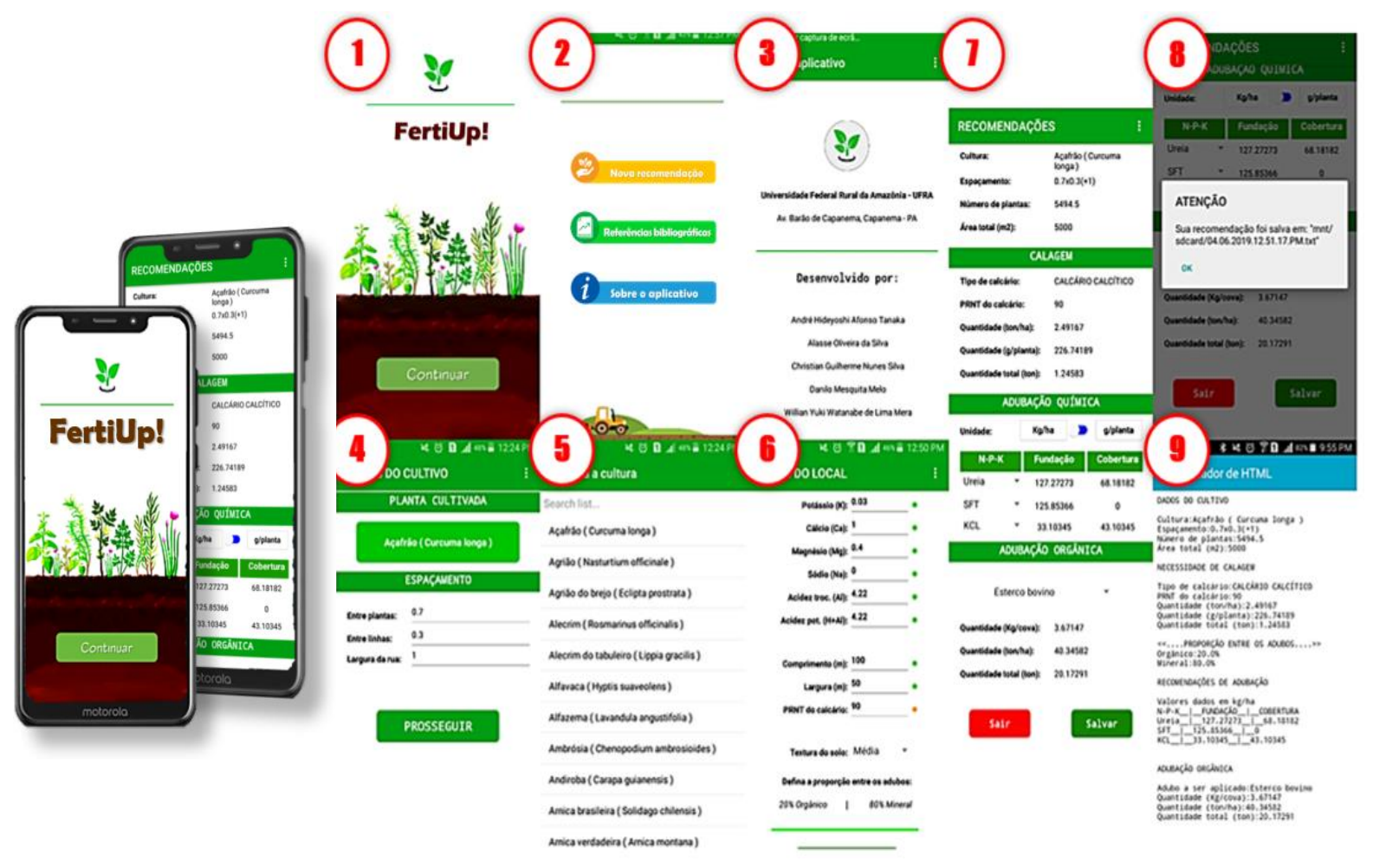




\section{Conclusões}

O aplicativo FertiUp! foi desenvolvido para que técnicos e produtores possam utilizálo em qualquer lugar, pois não carece de acesso à internet, possui uma linguagem simples e mostrou-se mais rápido, prático e eficiente na realização dos cálculos de calagem e adubação, em comparação com a forma tradicional (busca em livros, calculadoras e contas, manualmente, em papel), sendo perfeitamente capaz de otimizar o tempo de um assistente técnico ou mesmo do próprio agricultor. Portanto, este aplicativo constitui-se uma ferramenta valiosa no suporte às pessoas que lidam com cultivo de ervas medicinais, permitindo arquivar dados de recomendações anteriores e ter adubações mais precisas, evitando perdas econômicas e desbalanço nutricional no ambiente de cultivo.

\section{Referências}

ABDELAZIZ, Mohamed Ewis; POKLUDA, Robert; ABDELWAHAB, Mohamed Mahmoud. Influence of compost, microorganisms and NPK fertilizer upon growth, chemical composition and essential oil production of Rosmarinus officinalis L. Notulae Botanicae Horti Agrobotanici Cluj-Napoca, v. 35, n. 1, p. 86, 2007.

ABMRA. Agro mais jovem e conectado. 2018. Disponível em: http://www.abmra.org.br/2016/index.php/agro-mais-jovem-e-conectado/ Acesso em: 7 de junho de 2019.

AGUIAR, TEA; GONÇALVES, C.; PATERNIANI, MEAZG. Boletim 200: instruções agrícolas para as principais culturas econômicas. 2014.

AMARO, Geovani B. et al. Recomendações técnicas para o cultivo de hortaliças em agricultura familiar. Embrapa Hortaliças-Circular Técnica (INFOTECA-E), 2007. 
AMOROZO, M.C.M. A abordagem etnobotânica na pesquisa de plantas medicinais. Pp.47-68. In: L.C. Di Stasi (org.). Plantas medicinais: arte e ciência - Um guia de estudo interdisciplinar. São Paulo, Editora da Universidade Estadual Paulista, 1996.

ANTONIOLLI, L. R. Sistema de Produção de Uva de Mesa do Norte de Minas Gerais: Colheita e manuseio pós-colheita. Embrapa Uva e Vinho. ISSN, p. 1678-8761, 2005.

AZEVEDO, CD de; MOURA, MA de. Cultivo de plantas medicinais: guia prático. Niterói: Programa Rio Rural, 2010.

BERNARDI, R. "Vantagens de programar com Orientação a Objetos (OOP)". 2011. http://dev.rbtech.info/vantagens-de-programar-com-orientacao-a-objetos-oop/

CRAVO, M. S.; VIÉGAS, I. J. M; BRASIL, E. C. Recomendações de adubação e calagem para o Estado do Pará. EMBRAPA Amazonia Oriental, Bélem, PA (Brasil), 2007.

DUDA, Rodrigo et al. Elaboração de aplicativos para Android com uso do App Inventor: uma experiência no Instituto Federal do Paraná-Campus Irati. Revista Brasileira de Ensino de Ciência e Tecnologia, v. 8, n. 2, 2015.

GASSI, R. P. Bardana (Arctium lappa L.) cultivada sob diferentes doses de fósforo e camade-frango. Dourados, MS, 2006. 38 f. Dissertação (Mestrado em Produção Vegetal - Agronomia) - Universidade Federal de Mato Grosso do Sul.

GONÇALVES, O. “Por que Java?” In: DevMedia, https://www.devmedia.com.br/porquejava/20384. 2010.

KANTAR. Android vs. iOS. 2018. Webpage. Disponível em: https://www.kantarworldpanel.com/global/smartphone-os-market-share/ Acesso em: 07 de junho de 2019.

LACERDA，J. J. J.; SILVA，D. R. G. FERTILIZANTES ORGÂNICOS: USOS, LEGISLAÇÃO E MÉTODOS DE ANÁLISE. Boletim Técnico - n. o 96 - p.90. 2014.

LEÃO, Victor Miranda et al. Usos e benefícios das plantas em comunidades rurais de Capanema, Pará, Brasil. Cadernos de Agroecologia, v. 10, n. 3, 2016.

LEPSCH, Igo F. 19 lições de pedologia. Oficina de textos, 2016. 
LOPES, João Filipe Domingues. Cultivo e processamento de plantas aromáticas. 2014. Tese de Doutorado.

\section{MINISTÉRIO DA AGRICULTURA PECUÁRIA E ABASTECIMENTO. Hortaliças} PANCs atraem agricultores que querem diversificar produção de alimentos. 2019. Disponível em: http://www.agricultura.gov.br/noticias/hortalicas-pancs-atraem-a-atencao-deagricultores-que-querem-diversificar-producao-de-alimentos. Acesso em: 7 de junho de 2019.

MASSACHUSETTS INSTITUTE OF TECHNOLOGY - MIT. App Inventor 2. 2013. Webpage. Disponível em: http://appinventor.mit.edu/explore/about-us.html Acesso em: 7 de junho de 2019.

MONTEIRO, Silvia Marcela Ferreira et al. Análise do comércio de plantas medicinais no município de Capanema - Pará, Brasil. In: II cointer PDV-Agro 2017. Anais... 2017.

MOREIRA, A. et al. Diagnóstico Nutricional, Adubação e Calagem para Citros Cultivados no Estado do Amazonas (1. Aproximação). Manaus: Embrapa Amazônia Ocidental, 2008, 26 p. Embrapa Amazônia Ocidental. Documentos; 56.

OCAMPO, R.; VALVERDE, R. Manual de cultivo y conservación de plantas medicinales. TRAMIL, San José, Costa Rica, 2000.

OLIVEIRA JÚNIOR AC; FAQUIN V; PINTO JEBP. 2006. Efeitos de calagem e adubação no crescimento e nutrição de arnica. Horticultura Brasileira 24: 347-351.

PAPERT, S. Mindstorms: Children, Computers, and Powerful Ideas. Basic Books, New York, 1980.

POKRESS, Shaileen Crawford; VEIGA, José Juan Dominguez. MIT App Inventor: Enabling personal mobile computing. arXiv preprint arXiv:1310.2830, 2013.

RAIJ, B. Van; ANDRADE, J.C.; CANTARELlA, H.; QUAGGIO, J.A. (Ed.). Análise química para avaliação da fertilidade de solos tropicais. Campinas: Instituto Agronômico, 2001. 285p.

SARADHI, V. S. P. et al. Effect of NPK fertilizers on chemical constituents of Aloe vera leaves. Journal of Natural Remedies, v. 7, n. 2, p. 258-262, 2007. 
SILVA, Jonathan Braga et al. Do quintal à alimentação familiar: um estudo de caso baseado no espaço urbano produtivo e sua relação com a complementação da economia familiar. In: III cointer PDV-Agro 2018. Anais... 2018.

SBCS. Manual de adubação e de calagem para os Estados do Rio Grande do Sul e de Santa Catarina / Sociedade Brasileira de Ciência do Solo. Comissão de Química e Fertilidade do Solo. - 10. ed. - Porto Alegre, 2004.

SEBRAE. Tecnologia da Informação no Agronegócio. SEBRAE, 2017, https://view.officeapps.live.com/op/view.aspx?src=http://datasebrae.com.br/wpcontent/upload s/2017/11/TIC-no-Agro_-v5.pptx.

SOUZA, Carlos Alberto Martinelli et al. Crescimento em campo de espécies florestais em diferentes condições de adubações. Ciência Florestal, v. 16, n. 3, p. 243-249, 2006.

TAVARES, F. dos A.; SALAME, Marcos Filipe Alves; NASCIMENTO, R. da S. Aplicativo móvel para auxiliar ações de transferência de tecnologia de citros no Amazonas. In: Embrapa Amazônia Ocidental-Artigo em anais de congresso (ALICE). In: CONGRESSO BRASILEIRO DE AGROINFORMÁTICA, 10., 2015, Ponta Grossa. Uso de VANTs e sensores para avanços no agronegócio: anais. Ponta Grossa: Universidade Estadual de Ponta Grossa, 2015. Não paginado. SBIAgro 2015., 2015. 